\title{
Nuclear export signal (NES) of transposases affects the transposition activity of mariner- like elements Ppmar1 and Ppmar2 of moso bamboo
}

Muthusamy Ramakrishnan ${ }^{1}$, Ming-Bing Zhou ${ }^{1,2^{*}}$ (D), Chun-Fang Pan ${ }^{1}$, Heikki Hänninen ${ }^{1}$, Ding-Qin Tang ${ }^{1}$ and Kunnummal Kurungara Vinod ${ }^{3}$

\begin{abstract}
Ppmar1 and Ppmar2 are two active mariner-like elements (MLEs) cloned from moso bamboo (Phyllostachys edulis (Carrière) J. Houz) genome possessing transposases that harbour nuclear export signal (NES) domain, but not any nuclear localization signal (NLS) domain. To understand the functions of NES in transposon activity, we have conducted two experiments, fluorescence and excision frequency assays in the yeast system. For this, by sitedirected mutagenesis, three NES mutants were developed from each of the MLE. In the fluorescence assay, the mutants, NES-1, 2 and 3 along with the wild types (NES-0) were fused with fluorescent proteins, enhanced yellow fluorescent protein (EYFP) and enhanced cyan fluorescent protein (ECFP) were co-transformed into yeast system. To differentiate protein localisation under the NES influence, ECFP alone was fused to wild and mutant NES domains either on $\mathrm{N}$ - or C-terminal and not to EYFP. Fluorescence assay revealed that blue fluorescence of ECFP was more intense than the red fluorescence of the EYFP in the yeast cell matrix. Further, ECFP had a wider localisation in the cellular matrix, but EYFP was largely located in the nucleus. The NES-1 domain was related to the comparatively high spread of ECFP, while NES-2 and NES-3 indicated a low spread, implying that NES activity on nuclear export increased when the NES is made leucine-rich, while the signalling activity was reduced when the leucine content was lowered in the NES domain. In the transposon excision assay, the mutant and wild type NES of both the Ppmar elements were integrated into an Ade2 vector, and within the Ade2 gene. Co-transformation of the vector together with non-autonomous Ppmar transposons and NES-lacking transposases was used to assess the differential excision frequencies of the mutants NES domains. In both the MLES, NES-1 had the highest excision suppression, which was less than half of the excision frequency of the wild type. NES-2 and NES-3 elements showed, up to three times increase in transposon excision than the wild types. The results suggested that NES is an important regulator of nuclear export of transposase in Ppmar elements and the mutation of the NES domains can either increase or decrease the export signalling. We speculate that in moso bamboo, NESs regulates the transposition activity of MLEs to maintain the genome integrity.
\end{abstract}

Keywords: Mariner-like elements (MLEs), Nuclear export signal (NES), Transposase, Transposition activity, Ppmar1, Ppmar2, Moso bamboo

\footnotetext{
* Correspondence: zhoumingbing@zafu.edu.cn

'State Key Laboratory of Subtropical Silviculture, Zhejiang A\&F University, Lin'an, Hangzhou 311300, Zhejiang Province, People's Republic of China ${ }^{2}$ Zhejiang Provincial Collaborative Innovation Center for Bamboo Resources and High-efficiency Utilization, Zhejiang A\&F University, Lin'an, Hangzhou 311300, Zhejiang Province, People's Republic of China

Full list of author information is available at the end of the article
}

(c) The Author(s). 2019 Open Access This article is distributed under the terms of the Creative Commons Attribution 4.0 International License (http://creativecommons.org/licenses/by/4.0/), which permits unrestricted use, distribution, and reproduction in any medium, provided you give appropriate credit to the original author(s) and the source, provide a link to the Creative Commons license, and indicate if changes were made. The Creative Commons Public Domain Dedication waiver (http://creativecommons.org/publicdomain/zero/1.0/) applies to the data made available in this article, unless otherwise stated. 


\section{Background}

Transposable elements (TEs), or 'jumping genes' or transposons, are DNA sequences that have the ability to move within the genome [1]. Transposons are ubiquitous in plant and animal genomes in abundance. There are two distinctive types, (a) DNA transposons (class II) that transpose by a DNA-mediated "cut-paste" mechanism [2] and (b) retrotransposons (class I) that act through the "copypaste" mechanism involving an RNA intermediate [3]. There are several variants within each of the two types. One of the most prevalent DNA transposon families in eukaryotic genomes is the Tc1/mariner superfamily, which plays a significant role in genome evolution [4-6]. Because of its near-identical sequence similarity to the bacterial insertion sequence, IS630 [7], Tc1/mariner superfamily is expanded to include IS630 elements and is renamed as ITm (IS630-Tc1-mariner) superfamily. ITm transposons are characterized by self-driven mobility of its members and are generally independent of host factors to mediate transposition. In nature, they show a widespread distribution, frequent and total random insertions, and have a high frequency of heterologous transposition [8, 9]. Because of their versatile nature, they are used in genetic studies, as a tool in gene tagging, transgenesis and insertional mutagenesis [8, 9]. ITm transposons are further classified [10], among which three major families are, Tc1-like elements (TLEs) [11], mariner-like elements (MLEs) [12] and Pogo-like elements [13]. Among the MLEs, two important members, mosaic element 1 (Mos1) and Haematobia irritans mariner 1 (Himar1) [14] are widely studied for their cross genome transportability and used as tools in genetic studies. These hyperactive elements are found to increase their transposition activities when expressed in different host genomes such as bacteria by 200-800 [15] times, and by 10-50 times when mutated [16].

MLEs have a relatively simple structure, consisting mainly of terminal inverted repeats (TIRs) and an open reading frame (ORF) [17]. Their TIRs are generally 10-40 bp long and contain protein-binding elements. ORF is 1000-1500 bp long and encodes for the transposase (TPase) gene. TPases are enzymes responsible for transposition activity. Structurally, they contain a DNA binding domain and a catalytic domain [17]. Additionally, the TPase contains one or more short sequences of nuclear localization signals (NLS) [18] and nuclear export signals (NES) [19]. NES also is a short (8-15 residues) amino acid sequence consisting usually of four to five hydrophobic residues in a protein [20]. It is often leucine-rich [21]. Figuratively, TPase expression includes nuclear transcription and processing, mRNA export to the cytoplasm, protein synthesis, and protein folding and import back into the nucleus for mediating the transposition activity. Any change in these steps can affect the transposition frequency
[22]. While NLS acts for nuclear retention of the TPase molecules, NES mediates export out of the nucleus. Therefore, depending on the cellular stage and physiological state, counterbalancing activities of NLS and NES motifs can influence the transposition activity [16, 23]. Hancock et al. [24] found that a mutation of the NES domain in the TPase of mPing, a deletion derivative of autonomous rice PIF/ Harbinger transposon Ping, increased transposition activity in both yeast and plants. Similarly, Fattash et al. [19] identified that a mutation of a putative NES in the Ozma TPase dramatically increased transposition of the Eif and Goblin MITEs in yeast.

In our previous study, we reported an abundance of MLEs in various bamboo genomes belonging to 38 genera of the Bambusoideae family [25]. Consequently, two full-length $M L E$ transposons, named Ppmar1 and Ppmar2, were cloned from moso bamboo (Phyllostachys edulis (Carrière) J. Houz) genome [26]. Both of these transposons were demonstrated to transpose in yeast and Arabidopsis thaliana genome and exhibited their affinity towards TA-rich regions. They were also shown to get integrated into nearby genes [27-29]. TPases of both, Ppmar1 and Ppmar 2 harbour NES domains, but it is still uncertain whether and how the NESs affects their transposition. In order to examine this, we used sitedirected mutagenesis to mutate NES sequences of Ppmar1 and Ppmar2 in moso bamboo. The influence of the mutated NES domains on the localization of TPase in the cell and their transposition frequencies were systematically studied using different NES mutants by the help of a yeast screening system and yeast transposition assay. To the best of our knowledge, no earlier reports are available on the influence of NESs on TPase localization and transposition activity of Ppmar1 and Ppmar2 transposons.

\section{Methods \\ Prediction of NES sequences in Ppmar1 and Ppmar2 transposase}

The NES sequences of Ppmar1 and Ppmar2 TPase were predicted using NetNES 1.1 online software (http:// www.cbs.dtu.dk/services/NetNES/) [30] and were named Ppmar1-NES and Ppmar2-NES, respectively. The nucleotide sequences of Ppmarl and Ppmar2 TPases and their amino acid sequences are given in Additional file 1.

\section{Construction of NES domain with the ECFP fragments in the plasmid pPS1890}

In our earlier studies, full-length sequences of Ppmar1 and Ppmar2 were isolated from moso bamboo leaves and were cloned into pMD18-T vector [26-28]. In this study, we used these clones for amplifying Ppmar1 and Ppmar2. Using an overlap PCR, the NES sequences of the TPases were fused to the $\mathrm{C}$-terminal and $\mathrm{N}$-terminal 
of the enhanced cyan fluorescent protein (ECFP) $(730$ bp) of the pPS1890 plasmid DNA that also contained an NLS domain [31]. The constructed plasmids were named pPS1890-NES-ECFP (NES fused in the N-terminal of ECFP) and pPS1890-ECFP-NES (NES fused in the C-terminal of ECFP). The primer sequences used to amplify the ECFPNLS are given in Additional file 2.

\section{Mutations in NES domains of Ppmar1 and Ppmar2 transposases}

Based on the NES domain sequences of Ppmarl and Ppmar2, three types of NES domains were designed using NetNES 1.1 for mutating their amino acid sequences. This was done in order to obtain a strong NES signal (NES-1), a weak NES signal (NES-2), and an intermediate NES signal (NES-3) (Table 1). By the use of QuikChange Lightning Site-Directed Mutagenesis Kits (Stratagene, USA) TPase NES sequences in the plasmids pPS1890NES-ECFP and pPS1890-ECFP-NES were mutated to generate the three types of NES domains. All mutated sequences were sequenced for confirmation. The primer sequences used to mutate the NES sequences of TPases are given in Additional file 2. The mutated domains were designated as Ppmar1-NES-1, Ppmar1-NES-2, Ppmar1NES-3, Ppmar2-NES-1, Ppmar2-NES-2 and Ppmar2-NES3 . The wild types were correspondingly designated as Ppmar1-NES-O and Ppmar2-NES-O.

\section{Yeast ECFP fluorescence screening}

Two vectors, pPS1888 and pPS1890 (Addgene, Cambridge, USA), were used for yeast transformation. The pPS1888 contained an enhanced yellow fluorescent protein (EYFP), while the vector pPS1890 had ECFP. On transformation, yeast cells carrying pPS1888 emits a yellow-green fluorescence $(527 \mathrm{~nm})$ under $513 \mathrm{~nm}$ excitation, while the cells carrying the pPS1890 emits blue fluorescence $(475 \mathrm{~nm})$ under $433 \mathrm{~nm}$ excitation, both in the cell nucleus. Both of the vectors carry an NLS domain upstream of the fluorescent protein. In order to distinguish yellow-green fluorescence from blue fluorescence, yellow-green fluorescence was converted into red fluorescence using a Zeiss LSM 510 META laser scanning confocal microscope (Zeiss, Germany) [32].

The pPS1890 vector was recombined both with wildtype and mutant NES sequences. The recombined pPS1890 vector and the unrecombined pPS1888 vector were transformed into yeast. After incubating at $30^{\circ} \mathrm{C}$ for 10 days, a single colony was selected for fluorescence observations by a Zeiss LSM 510 META laser scanning confocal microscope. The individual channels of ECFP (blue) and EYFP (red) were merged to produce the final images using Zeiss LSM Software ZEN 2009 of a confocal microscope. The intensity of both blue and red fluorescence was quantified in each channel of ECFP and EYFP, respectively using ImageJ v.1.5.2a [33]. The red fluorescence emitted by the pPS1888 vector is known to occur in the nucleus, so based on the relative localization of blue and red fluorescence, the distribution of the modified ECFP in cells was determined.

\section{Construction of pAG413gal-Tpase1 and pAG413gal- Tpase2 vectors having NES sequences}

TPase sequences of Ppmar1 and Ppmar2 containing NES sequences were amplified with Not I and EcoR V sites added. The amplification fragments were digested by Not I and EcoR V enzymes. The pAG413gal-ccdB vector with His3 selectable marker was also cut by both restriction enzymes, and the big fragment was recovered. Then the digested TPase fragments and the backbone of pAG413gal-ccdB were ligated by $\mathrm{T}_{4}$ DNA ligase, resulting in the recombined vectors pAG413gal-Tpase 1 and pAG413gal-Tpase2. The TPase was promoted to be expressed under the gal promoter. Using QuikChange Lightning Site-Directed Mutagenesis Kit the NES sequences in the TPase sequences of pAG413gal-Tpase1 and pAG413gal-Tpase2 were mutated into three

Table 1 Amino acid mutations in NES domains of Ppmar1-NES and Ppmar2-NES transposases of moso bamboo

\begin{tabular}{|c|c|c|c|c|}
\hline Names of NES & NES type & Sequence & Mutated site & $\begin{array}{l}\text { Approximate } \\
\text { NES score }\end{array}$ \\
\hline Ppmar1-NES-0 & Wild type NES & KQRLEREDRLPLQIP & - & 0.9 \\
\hline Ppmar1-NES-1 & Strong NES & KLRLEREDRLPLQIP & $\mathrm{Q} \rightarrow \mathrm{L}$ & 1 \\
\hline Ppmar1-NES-2 & Weak NES & KQRLEREDRAPAQIP & $\mathrm{L} \rightarrow \mathrm{A} ; \mathrm{L} \rightarrow \mathrm{A}$ & 0 \\
\hline Ppmar1-NES-3 & Intermediate NES & KQRLEREDRAEIQIP & $\mathrm{L} \rightarrow \mathrm{A} ; \mathrm{P} \rightarrow \mathrm{E} ; \mathrm{L} \rightarrow \mathrm{I}$ & 0.5 \\
\hline Ppmar2-NES-O & Wild type NES & RNGVLSIRLQCDL & - & 0.85 \\
\hline Ppmar2-NES-1 & Strong NES & RLGVLSIRLQCDL & $\mathrm{N} \rightarrow \mathrm{L}$ & 1 \\
\hline Ppmar2-NES-2 & Weak NES & RNGVLSARLQCDL & $\mathrm{I} \rightarrow \mathrm{A}$ & 0 \\
\hline Ppmar2-NES-3 & Intermediate NES & RNGVLLIRLQCDL & $S \rightarrow L$ & 0.5 \\
\hline
\end{tabular}

The bold red letters stand for mutated amino acids

Letters on the left- and right-hand sides of the arrows indicate the wild type and the mutated amino acid, respectively. The NES types were identified based on the NES scores

$Q$ glutamine, $L$ leucine, $A$ alanine, $P$ proline, $E$ glutamic acid, $N$ asparagine, $I$ isoleucine, $S$ serine 
versions corresponding to the three NES types (Table 1). The primer sequences are provided in Additional file 2.

\section{Construction of non-autonomous pWL89A-Ppmar1NA and pWL89A-Ppmar2NA vectors}

The pMD18-T vector [28] containing full-length Ppmar1, including two target site duplicates (TSDs) of dinucleotide Thymine ( $\mathrm{T}$ ) and Adenine (A), was cut by BseR I and the resultant 5' and 3' TIRs of Ppmar1 and their adjacent sequences were ligated together leading to truncated Ppmar1NA. The Ppmar1NA was 778 bp long consisting of the TIRs, TSDs and the sub-terminal sequences without TPase. The sequence of Ppmar1NA is indicated in Additional file 3. Simultaneously, the non-autonomous transposon of Ppmar2, Ppmar2NA, was also constructed (Additional file 3). The $5^{\prime}$ and $3^{\prime}$ TIRs and their adjacent sequences of Ppmar2 were amplified. The details of the primers (Mini-s-1-20-1F and Mini-s-1-20-1R1) used for amplifying the 5'TIR region and the primers (Mini-s-1-202F1 and Mini-s-1-20-2R) used for amplifying the 3'TIRs region are given in Additional file 4. Combining the resultant fragments amplified by the upstream primer (Mini-s-1-20-1F) and the downstream primer (Minis-1-20-2R), resulted in the Ppmar2NA. The sequence of Ppmar2NA is indicated in Additional file 3.

Ppmar1NA and Ppmar2NA were inserted into the Xho I site at the $5^{\prime}$ untranslated region (UTR) of the Ade2 gene in the vector pWL89A possessing two selectable markers of Ura 3 and Ade2. This resulted in two recombined vectors, named pWL89A-Ppmar1NA and pWL89A-Ppmar2NA, respectively.

\section{Yeast transposition assay of excision frequencies}

In order to quantify the transposon excision frequency (TEF) catalyzed by mutated TPases in the yeast cells, six separate yeast transposition assays were performed. Both of the pairs of vectors (pAG413gal-Tpase1 and pWL89APpmar1NA; pAG413gal-Tpase2 and pWL89A-Ppmar2NA) were transformed into yeast strain DG2523 (MAT-alpha Ura3-167 Trp1-HisG Leu2-HisG His3-Del200 Ade2-HisG). After double transformation, yeast strains were grown on a complete supplement mixture (CSM) medium lacking histidine and uracil (CSM-his-ura) with $2 \%$ galactose at $30^{\circ} \mathrm{C}$ for 10 days. Single colonies were then dissolved in $150 \mu \mathrm{l}$ water and plated on a medium lacking adenine, histidine and uracil (CSM-ade-his-ura) and having $2 \%$ galactose as the sole carbon source. The plates were incubated at $30^{\circ} \mathrm{C}$ for 20 days to allow the growth of revertant colonies.

In the yeast assay $A d e 2$, revertant frequencies were counted for the wild type and the NES mutated TPase constructs. In detail, the cells in each galactose-induced colony were suspended in $50 \mu \mathrm{l}$ of water and plated on a media lacking adenine. Growth of the yeast cells on medium lacking adenine required the excision of transposon and the expression of the Ade 2 gene. An equal volume, but diluted $1 \times 10^{5}$ times from the cell suspension mentioned above, was placed on CSM-adehis-ura medium to obtain the total number of viable cells in the galactose-induced colony. Six separate experiments were carried out and the excision frequencies obtained from them were averaged in the analysis of the results.

\section{Results}

Prediction of NES of Ppmar1 and Ppmar2 transposases

The NES sequences of TPases were predicted to be located at the positions at $471-481 \mathrm{bp}($ score $=0.90)$ for Ppmar1 and 435-447 bp for Ppmar2 (score $=0.85$ ). They had sequence lengths of $120 \mathrm{bp}$ and $126 \mathrm{bp}$ respectively with amino acid sequences of KQRLEREDRLPLQIP and RNGVLSIRLQCDL. Both the NES domains were leucine-rich with constituent hydrophobic amino acids such as isoleucine (I), proline (P), glycine $(\mathrm{G})$, valine $(\mathrm{V})$ and leucine $(\mathrm{L})$. Evaluated by the software NetNES 1.1, three types of NES domains were predicted for mutation with strong, intermediate and weak transport signals and having predicted scores close to 1.0, 0.5 and 0.0, respectively. Details of amino acid mutations and scores are given in Table 1 . The NES scores close to 1.0 (Ppmar1-NES-1 and Ppmar2$N E S-1)$ are considered to have strong regulation of TPase export from the nucleus. The intermediate NES scores close to 0.5 (Ppmar1-NES-3 and Ppmar2-NES-3) are considered to have a moderate level of ex-nuclear export while the signal scores close to 0.0 (Ppmar1NES-2 and Ppmar2-NES-2) are considered to have a weak regulation.

\section{Differential fluorescence in mutant yeast cells}

In all the cases, irrespective of the NES used, the yellow fluorescence emitted by the EYFP as detected by the red colour in laser scanning confocal microscopy was limited to the nucleus alone. The localization of different versions of NES fused to the ECFPs in the yeast cells were detected in the fluorescence screening system. In both of the wildtypes, Ppmar1-NES-O and Ppmar2-NES-O, blue fluorescence was observed both in the nucleus and the cytoplasm, with the former displaying relatively stronger fluorescence than the latter. However, in the mutant types with weaker export signals, Ppmar1-NES-2 and Ppmar2-NES-2, the blue fluorescence was only observed in the nucleus and not in the cytoplasm (Figs. 1, 2, 3 and 4). Whereas, in Ppmar1NES-1 and Ppmar2-NES-1, the mutants with stronger NES, a deeper blue fluorescence was observed in the cytoplasm than in the nucleus. In the intermediate mutant types, Ppmar1-NES-3 and Ppmar2-NES-3, the blue fluorescence was rather uniformly distributed in the nucleus and the cytoplasm (Figs. 1, 2, 3 and 4). 


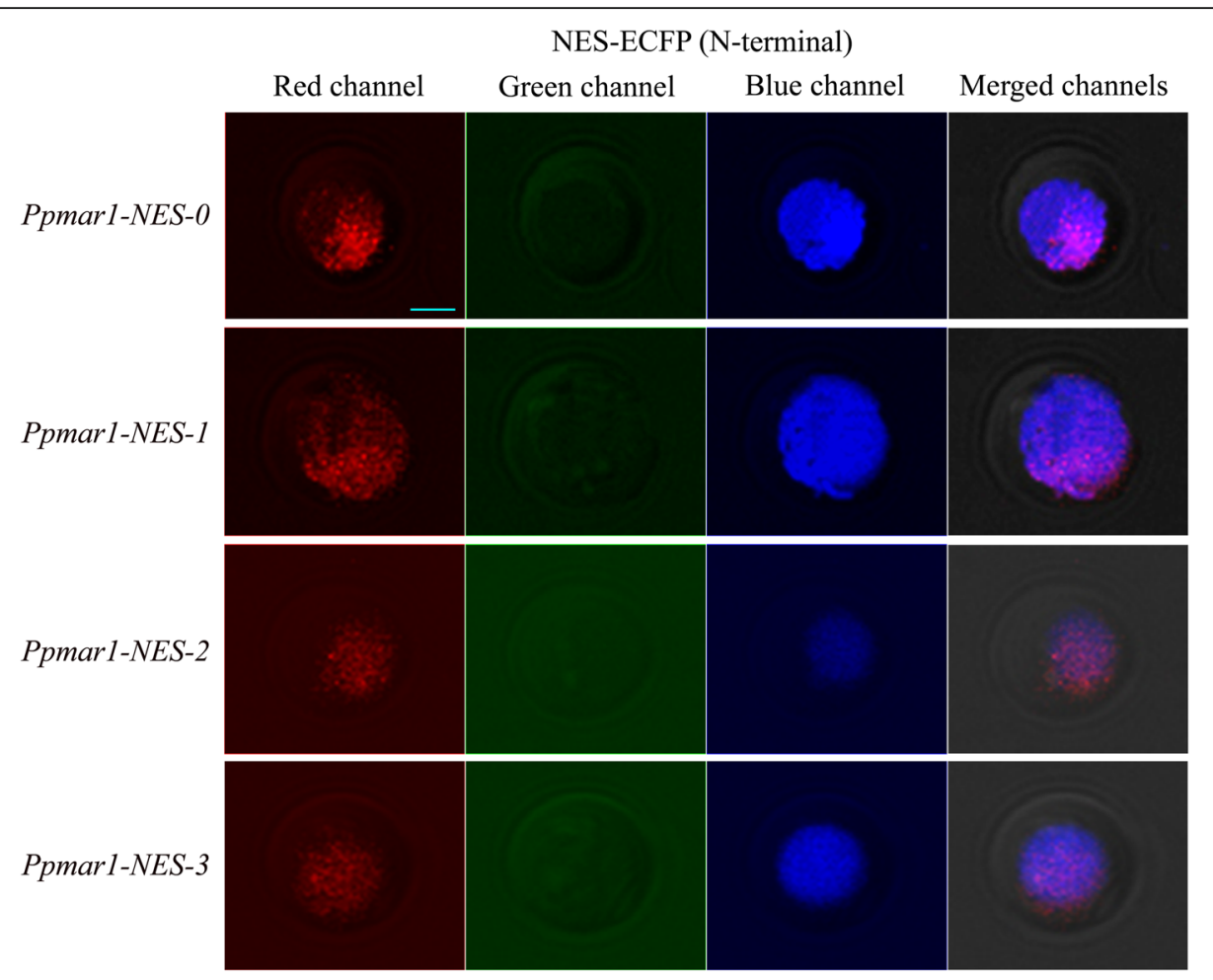

Fig. 1 The localization signal of reporter proteins fused to various Ppmar1-NES transposase in yeast. Fluorescence observation of ECFP fused with NES of Ppmar 1 transposase in N-terminal (NES-ECFP). The photographs illustrate the microscopy of the red channel, green channel and blue channel and merged channels of yeast expressing ECFP fused into different versions of NES. The nuclear translocation EYFP (yellow-green) was co-expressed to provide a nuclear marker. In order to differentiate yellow-green fluorescence from blue fluorescence, yellow-green fluorescence was converted into red fluorescence. Scale bar indicates $5 \mu \mathrm{m}$

\section{Quantification of fluorescence intensity}

The mean pixel intensity of blue fluorescence (45.6) of ECFP fused with various Ppmar1-NES and Ppmar2-NES TPases was almost two times higher than red fluorescence (23.2) emitted by the nuclear marker protein EYFP (Fig. 5). The fluorescent intensity showed no apparent difference with the position of NES attachment to the fluorescence proteins. The expression of red fluorescence was more or less consistent (range of 17.6 to 30.5) in all Ppmar1-NES and Ppmar2-NES TPases including wild and mutants, whereas the expression of blue fluorescence varied widely (range of 25.6 to 66.0). Among the wild types, Ppmar1-NES-O showed a higher intensity of blue than Ppmar2-NES-O. In both the high-affinity mutants, Ppmar1-NES-1 and Ppmar2-NES-1, the mean blue fluorescence intensity was higher than other mutants. However, in the low-affinity mutants, Ppmar1-NES-2 and Ppmar2-NES-2, the pixel intensity was lower than the intermediate affinity mutants, Ppmar1-NES-3 and Ppmar2-NES-3, but only when the NES was attached to the N-terminal end. When attached to the C-terminal end of the fluorescent proteins, Ppmar1-NES-3 showed lower intensity than Ppmar1-NES-2 (Additional file 5).
The influence of mutated NES on the transposon excision frequency

The TEFs by both Ppmar1 and Ppmar2 varied conspicuously between wild-type and the mutants that carried variation only in the NES domain (Fig. 6a and b). The non-autonomous mutants (Ppmar1NA and Ppmar2NA) catalyzed by the NES-2 mutants of both Ppmarl and Ppmar2 showed 300 and $200 \%$ higher excision frequency respectively than by the wild-types, Ppmar1-NES-O and Ppmar2-NES-O. Interestingly, the TEFs of Ppmar1NA and Ppmar2NA catalyzed by NES-1 mutants were respectively about 45 and $40 \%$ of that the wild-type TPases. The NES-3 mutants, however, showed 320 and $150 \%$ of the TEFs for Ppmar1NA and Ppmar2NA, in that order (Fig. 6a and b).

\section{Discussion}

The moso bamboo MLEs, Ppmar1 and Ppmar2, are active DNA transposons. The TPase enzymes of these elements show significant sequence analogy indicating their active functionality. The interesting fact that the TPase contains an NES domain and no NLS domain, signifies a potential biological role for these transposons. 


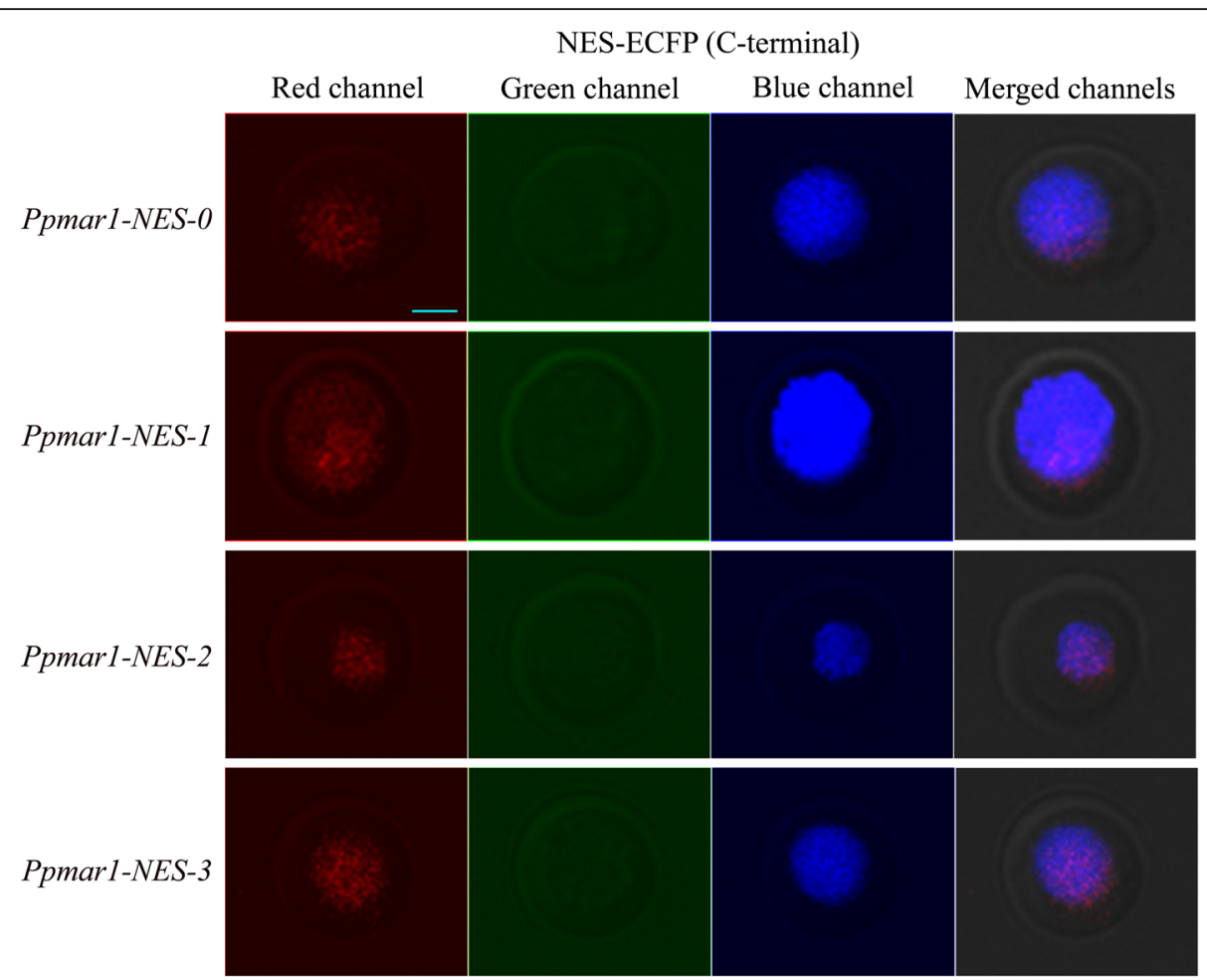

Fig. 2 The localization signal of reporter proteins fused to various Ppmar1-NES transposase in yeast. Fluorescence observation of ECFP fused with NES of Ppmar1 transposase in C-terminal (ECFP-NES). The photographs illustrate the microscopy of the red channel, green channel and blue channel and merged channels of yeast expressing ECFP fused into different versions of NES. The nuclear translocation EYFP (yellow-green) was co-expressed to provide a nuclear marker. In order to differentiate yellow-green fluorescence from blue fluorescence, yellow-green fluorescence was converted into red fluorescence. Scale bar indicates $5 \mu \mathrm{m}$

Although NESs have been identified in eukaryotic TPase [24], their biological functions are not yet known. However, it is well known that NESs acts in the import of transposases into the cytoplasm, thereby suppressing their excision activity. In the current study, we for the first time attempted to examine the effect of NES domain in the excision activity of Ppmar1 and Ppmar2 and their potential role in controlling the transposition in the event of site-specific mutations.

The NESs are assumed to have an unusually significant role in moso bamboo transposons because of the absence of an NLS domain, which acts as a counterbalancing system. NLS acts in importing the TPase after synthesis from the cytoplasm to the nucleus, to incite transposition [34], while the NES facilitates export back to the cytoplasm. Therefore, a clear balance of NLS and NES activities is essential for transposon activity. Accordingly, in moso bamboo, we can assume that transposition could be largely dependent on the NES function. Since the NES actively returns the TPases back to the cytoplasm, the TPase residence time in the nucleus is reduced, thereby suppression of transposition is achieved in the resident genome.
The amino acid sequences in the NES domains are believed to play a potential role in export signalling. Our TPase mutant constructs having different export potentials in their NES domains could suggest a role for certain amino acids, such as leucine, in nuclear export signalling. Further, we have studied the activity in a yeast system for two main reasons, the first being the absence of a DNA transposon in yeast, a unique feature among all eukaryotic organisms [35] and the second being the small size of the yeast genome and its well-known genetics [36]. Additionally, the Ppmar transposons were successfully activated in a yeast system in earlier studies [28].

\section{Differential cellular localization of NES domains}

In the yeast fluorescence screening system used in the present study to display the cellular localization of the NESs, the fusion to fluorescent proteins could be efficiently located using confocal microscopy. The choice of fluorescent proteins as the marker for subcellular localization owes to their easy detection in the living cells. Two fluorescence signal vectors were used, pPS1888 and pPS1890, which harboured EYFP and 


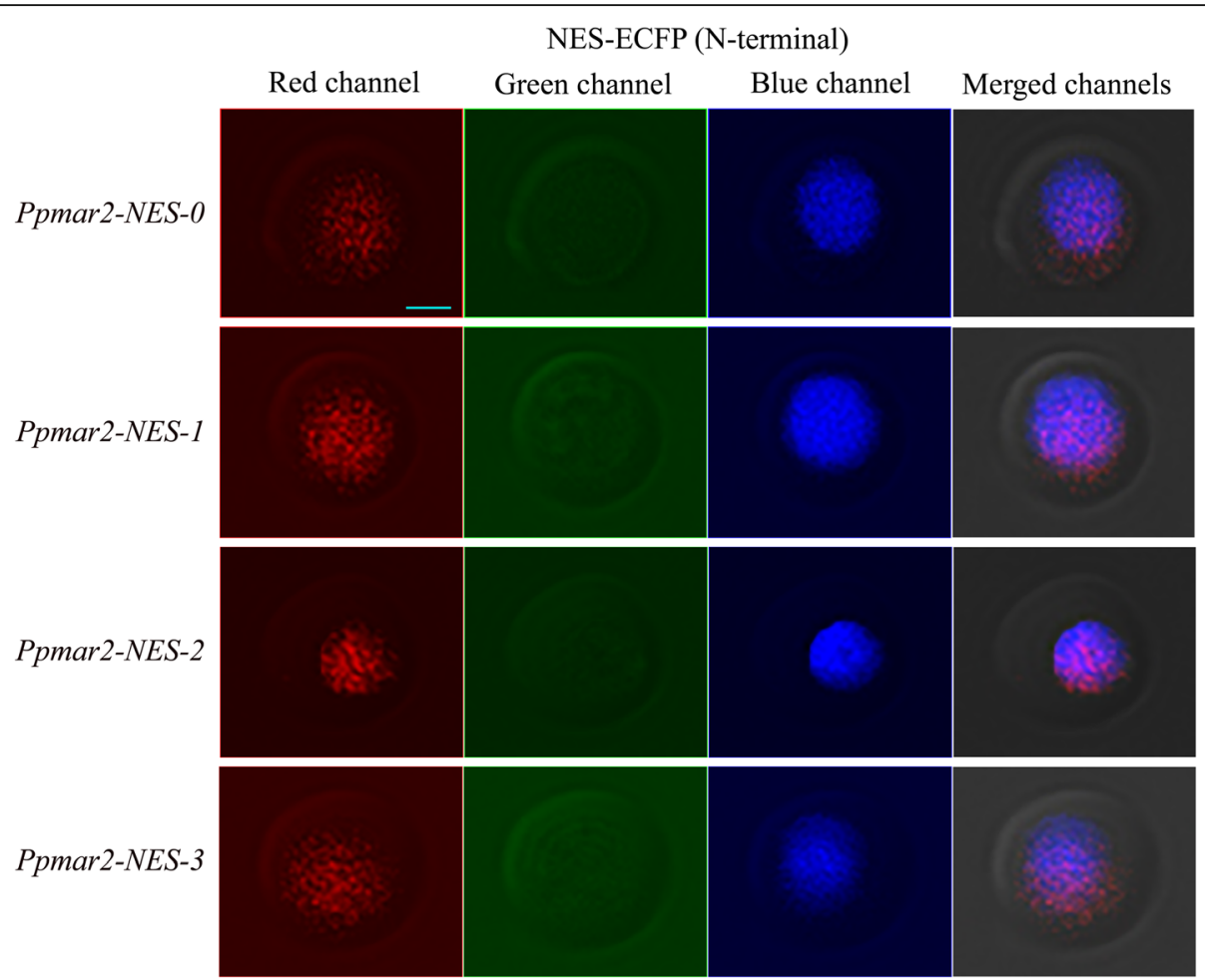

Fig. 3 The localization signal of reporter proteins fused to various Ppmar2-NES transposase in yeast. Fluorescence observation of ECFP fused with NES of Ppmar2 transposase in N-terminal (NES-ECFP). The photographs illustrate the microscopy of the red channel, green channel and blue channel and merged channels of yeast expressing ECFP fused into different versions of NES. The nuclear translocation EYFP (yellow-green) was co-expressed to provide a nuclear marker. In order to differentiate yellow-green fluorescence from blue fluorescence, yellow-green fluorescence was converted into red fluorescence. Scale bar indicates $5 \mu \mathrm{m}$

ECFP, respectively [37]. Since there was an NLS domain upstream from both of the fluorescent proteins in the vector, there could be an enhanced tendency of exclusive localization of both of the proteins in the nucleus. In order to differentiate their localization tendencies regulated exclusively by the presence of NLS domains, we fused NES domains only to ECFP and not to EYFP. Therefore, in the yeast system after a co-transformation of both of the vectors, we expected EYFP to remain in the nucleus, but ECFP can be either in the nucleus or in the cytoplasm, or in both because the NES domains could get ECFP exported to the cytoplasm. The localization of the fluorescent proteins was judged by the colour emitted by them in the yeast cells. The red fluorescence indicated the presence of EYFP and blue that of ECFP, and the intensity of fluorescence could be attributed to the density of the protein. When mutated NESs have differential nuclear export potential, it could affect the distribution of ECFP between nucleus and cytoplasm.

In our assays, we could see less EYFP fluorescence (red colour) than ECFP fluorescence (blue colour) in the yeast cells. The EYPF were mostly concentrated towards the nuclear region irrespective of the variation in the
NES domains in the co-transformed vector, pPS1890. We assumed that preferential localisation EYFP in the nucleus could be driven by the NLS domain, since EYPF was not fused to NES, unlike that of ECFP. Additionally, the intensity of the red fluorescence remained within a narrow range in all the co-transformed systems including wild and mutants, and irrespective of the fact that the NES was attached to $\mathrm{N}$ - or C-terminal end of the ECFP protein in the co-transformed vector. This confirmed that the presence of EYPF was not under the influence of NES domains used in the experimental systems.

Further ascertaining the role of NES domains in the distribution of fluorescent protein, we could observe variation in the presence of blue fluorescence from ECFP in both the nucleus and the cytoplasm, although distinct quantification between these could not be done. When the wild type NES was fused to the protein, there was a high intensity of blue colour across the cell but the red colour was located only at the nuclear regions in all the four systems, that involved two different Ppmar elements (Ppmar1 and Ppmar2) and two different fusion systems (N-terminal and $\mathrm{C}$-terminal). This suggested that the fluorescent proteins fused to NES domains had 


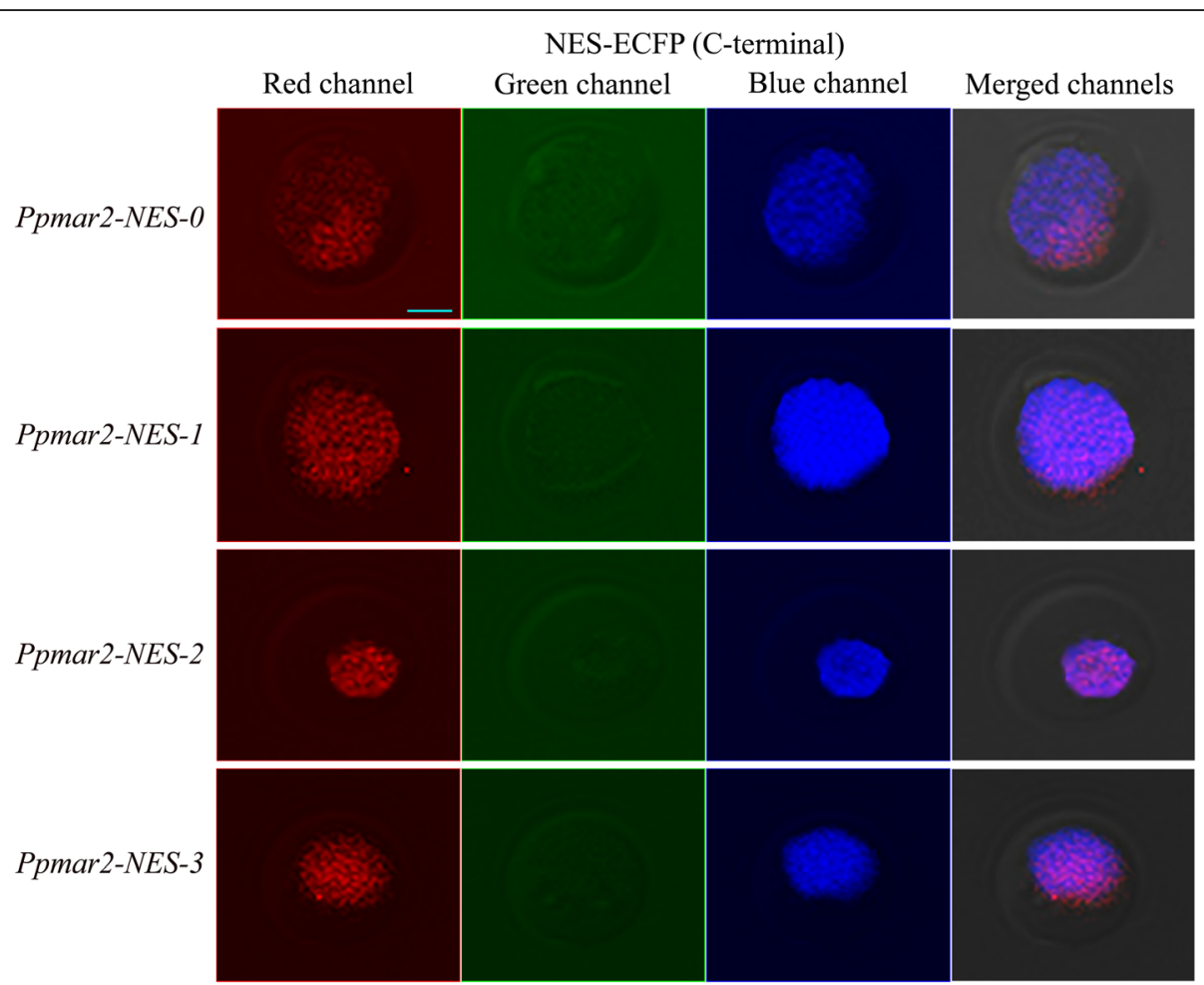

Fig. 4 The localization signal of reporter proteins fused to various Ppmar2-NES transposase in yeast. Fluorescence observation of ECFP fused with NES of Ppmar2 transposase in C-terminal (ECFP-NES). The photographs illustrate the microscopy of the red channel, green channel and blue channel and merged channels of yeast expressing ECFP fused into different versions of NES. The nuclear translocation EYFP (yellow-green) was co-expressed to provide a nuclear marker. In order to differentiate yellow-green fluorescence from blue fluorescence, yellow-green fluorescence was converted into red fluorescence. Scale bar indicates $5 \mu \mathrm{m}$

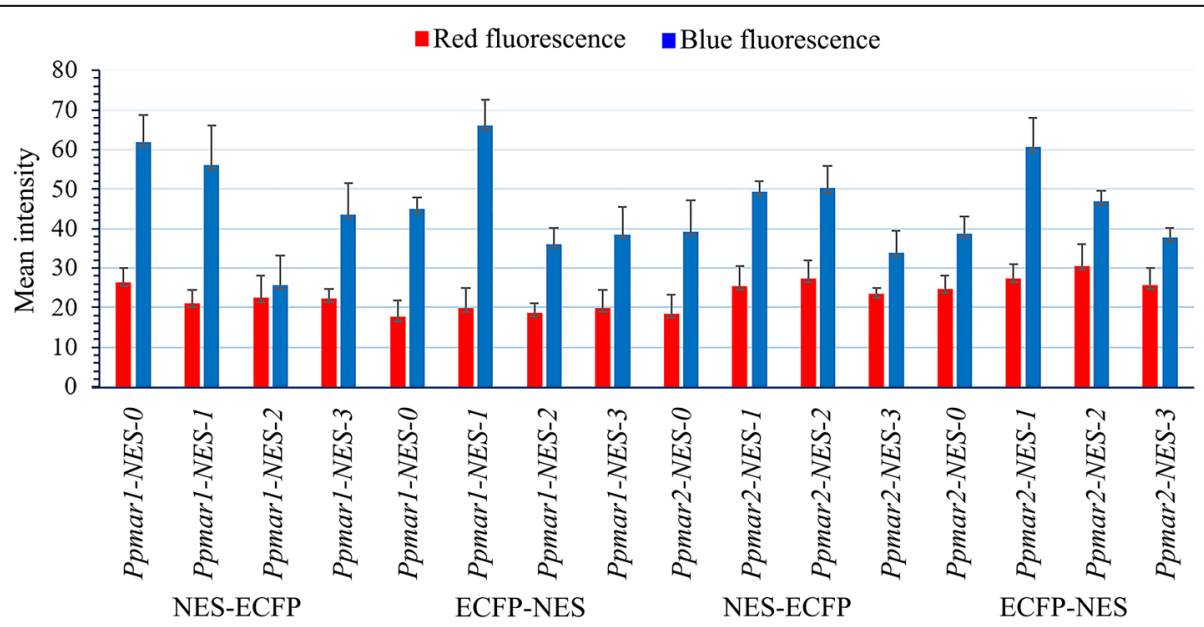

Fig. 5 The mean intensity of fluorescence of reporter proteins fused to various Ppmar1-NES and Ppmar2-NES transposase in yeast. Blue fluorescence is ECFP fused with various NES of Ppmar1 and Ppmar2 transposase in N-terminal (NES-ECFP) and C-terminal (ECFP-NES). Red fluorescence is EYFP (red) was co-expressed to provide a nuclear marker 

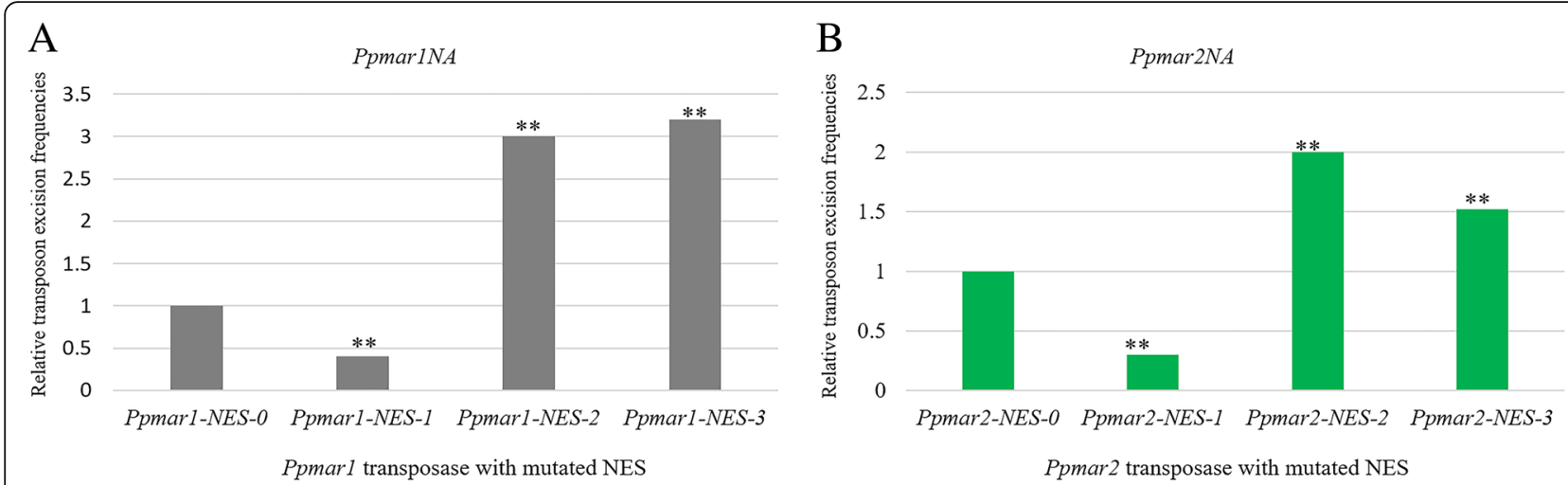

Fig. 6 a Transposition frequencies of Ppmar1NA catalyzed by Ppmar1 transposase with mutated NES. $\mathbf{b}$ Transposition frequencies of Ppmar2NA catalyzed by Ppmar2 transposase with mutated NES. The vertical axis indicates the ratio of the mean of excision frequency catalyzed by $P p m a r 1$ and Ppmar2 transposase mutants in six independent experiments, to the excision frequency catalyzed by the wild-type Ppmar1 and Ppmar2 transposase. Statistical significance of the differences: ${ }^{* *}$ very significant $(P<0.01)$

higher mobility across the cell matrix, which could be due to their active export from the nucleus to the cytoplasm. Further, a relatively wider spread of the blue fluorescence across the yeast cell suggested that the influence of the NES domain on the export process was relatively stronger than the retention effect of the NLS domain. Reiterating the role of NES on the intracellular mobility of the ECFP, a restricted spread of blue colour was noticed when the NES was mutated to have weaker signalling (NES-2), wherein both blue and red fluorescence was found confined to the nuclear region alone. $N E S$ - 2 mutants had their leucine and isoleucine mutated to alanine, thereby losing the signalling property. This implied that altering the leucine level can knock out their functional potential since NES domains are generally leucine-rich [38]. This hypothesis was further supported by the NES-1 mutation, which showed a stronger blue fluorescence in the cytoplasm in most of the systems except for Ppmar2-NES-1 fused on the C-terminal. In NES-1 mutation, glutamine and asparagine were mutated to leucine, increasing the leucine richness. Nevertheless, when there was no change in the leucine richness as in NES-3 mutants, uniform blue fluorescence could be observed from both the nucleus and the cytoplasm. Among the NES-3 mutants, in Ppmar1-NES-3, leucine was mutated to alanine and isoleucine, while proline was changed to glutamic acid; whereas in Ppmar2-NES-3 serine was mutated to leucine. This could possibly render the NES-3 mutants to intermediate signalling domains thereby bringing a balance in the protein distribution across the cell matrix. These observations from the cellular localization of the fluorescent proteins fused to NES of the TPases are suggestive of a parallel active role of the transposases in moso bamboo transposons Ppmarl and Ppmar2. Furthermore, there was no difference in export activity with respect to the site in which NES were fused to ECFP, either on C-terminal or N-terminal. Furthermore, the intensity of blue fluorescence observed for all of the mutants corresponded well with the signal values of the mutants predicted by the software, NetNES 1.1. as strong, weak, or intermediate.

\section{Excision of the transposons in yeast}

In the excision frequency assay, yeast colonies can develop in Ade minimal medium only if the Ade 2 gene is expressed. Since Ade2 is silenced in the vector by placing a non-autonomous MLE within the gene, excision of the transposon is essential for the Ade2 expression. Therefore, the development of revertant yeast colonies can be directly associated with the rate of excision of the non-autonomous transposon from the Ade 2 gene. Since the excision is dependent on the TPase activity, the colony development can be further related to the Tpase efficiency which in turn is associated with the NES domain which is mutated. Although we have not directly observed transposition of moso bamboo MLEs in the present study, because we have not observed the insertion, from the transposition frequency assays for both Ppmar1 and Ppmar2, we could gather the efficiencies of NES domains in both wild type and mutants in excising the transposon from the yeast genome. Since excision is an integral part of the transposition activity of the MLEs, the excision assay would, therefore, provide us with indirect evidence of transposition.

Among the NES mutants, we could find that NES-1 mutants had produced very few revertant colonies than other systems, indicating that NES-1 domains had relatively less amount of TPases in the nucleus to incite excision process. This also implied that TPases produced in the co-transformed yeast cells could have been exported out of the nucleus by the increased signalling 
activity of the NES-1 domain. Therefore, NES-1 domains were more than two times efficient than the wild type in preventing transposon excision. On the contrary, both NES-2 and NES-3 mutants had poor nuclear export signalling, due to which there was significantly high TPase activity in the nucleus and increased in transposon excision. This suggested that NES-2 and NES-3 mutants could have facilitated a relatively long residence time for the TPases in the nucleus. In the Ppmar1-NES3 mutant (L477A-P478E-L479I), the excision frequency was higher than Ppmar1-NES-1 and Ppmar1-NES-2. However, among the Ppmar2-NES, excision frequency of NES-3 mutant was lower than NES-2 mutant.

The possible role of NESs of moso bamboo MLEs, Ppmar1 and Ppmar2 are to reduce their transposition activity. This can easily be illustrated from the fact that TPases are translated and produced in the cytoplasm and exported into the nucleus to bind to the target elements within the genome. However, with the weakening of the NES motif by mutation, the export machinery of TPases to the cytoplasm fails, and result in the nuclear accumulation of TPases, thereby triggering transposition [24]. Since the TPases are essential for the MLE transposition, their residence time in the nucleus could be an important factor determining the transposition frequency. A relatively long residence time would offer an increased chance of a TPase to bind to TIRs and excise the transposons [24]. Therefore, NES could be playing a significant role in controlling the residence time of TPase. In the rice MLE, Ping and Pong, which also possesses TPases harbouring NES domain [24], mutation of the NES domain was reported to have increased excision and transposition activity in rice and Arabidopsis. The mutant of Ping (mPing) had an increased activity by about six times when the Ping TPase-NES was weakened by mutation (L384/386A), while the Pong mutant had an increased excision and transposition activity by about 16 times following an NES mutation (L418/420A) [24]. In the same way, the NES mutant of Ozma TPase had resulted in an increased transposition activity of at least 4160 times for the Eif and Goblin MITEs in yeast [19].

By using non-autonomous mutants of Ppmar1 and Ppmar2, we have decisively demonstrated that NES domains could regulate the transposon excision activity by regulating the nuclear export of TPase. The TEFs of all the NES mutants showed precise agreement with the NES export activity as observed in the fluorescence screening system. The activity of the wild type NES suggested that keeping the TPases less resident in the nucleus can prevent any undesired transposition activity, thereby minimizing any unwarranted genetic changes in the system so that the genome integrity could not be compromised. Since there is no NLS domain in the moso bamboo MLEs, we conclude that NES prominently regulates the transposition activity of these elements. Our results further suggest that TPases harbouring leucine-rich NESs domains are more efficient in maintaining genome integrity than those harbouring leucine-poor NES. Furthermore, to validate the hyperactivity of mutated NESs, they needed to be tested in the bamboo genome. This could help in developing active NES domains for tools of genetic manipulations and bamboo breeding.

\section{Conclusion}

In conclusion, NES is an active domain in the Ppmar1 and Ppmar2 TPases of moso bamboo and the mechanism of NES is highly specific to the TPases. The present study has evidenced a significant function of the NES domain in the nuclear export of transposase, which could be important in maintaining genome integrity. Although NES domains are common in various genetic systems and mediate crosstalk between the cytoplasm and the nucleus either by interacting with signalling molecules or by their own movement [39, 40], there is no evidence any other additional functions to NES of moso bamboo MLEs other than controlling transposition. From the evidence, we speculate that NES could act as a regulatory switch to control the export of TPase and thereby control the transposon activation. We have also demonstrated that the NES domain itself can be easily be mutated to bring in changes in the nuclear export signalling. Therefore, we further speculate that the NES domain might have a function of maintaining the genome integrity under favourable conditions. On the contrary, the onset of unfavourable conditions could alter its function to create more mutations to increase cellular plasticity. Notwithstanding, these speculations of broader biological functions of NES needs further investigations, as conclusive evidence are still missing. TEs are abundant in the moso bamboo, and they play a major role in the genome evolution. Since transposon activity has evolutionary relevance, it would be interesting to understand the role of NES in genome evolution, spontaneous mutations and ultimately deciding the fitness and plasticity of the genome under selection pressure.

\section{Additional files}

Additional file 1: The nucleotide sequences of Ppmar1 and Ppmar2 transposases and their amino acid sequences. (DOCX $18 \mathrm{~kb}$ )

Additional file 2: The sequences of primers used in the study to amplify the enhanced cyan fluorescent protein (ECFP) with a nuclear localization signal (NLS) (ECEP-NLS) and to mutate the NES sequences of Ppmar1 and Ppmar2 transposases. (DOCX $17 \mathrm{~kb}$ )

Additional file 3: The nucleotide sequences of Ppmar1NA and Ppmar2NA transposons (non-autonomous). (DOCX $15 \mathrm{~kb}$ ) 
Additional file 4: The sequences of primers used in the study for amplification of Ppmar1NA, Ppmar2 and Ppmar2NA transposons. (DOCX $16 \mathrm{~kb}$ )

Additional file 5: The fluorescence intensity of both blue and red colour of ECFP and EYFP channels, respectively. (DOCX $18 \mathrm{~kb}$ )

\section{Abbreviations}

ECFP: Enhanced cyan fluorescent protein; EYFP: Enhanced yellow fluorescent protein; MLEs: Mariner-like elements; NES: Nuclear export signal; NLS: Nuclear translocation signal; ORF: Open reading frame; TEFs: Transposon excision frequencies; TEs: Transposable elements; TIRs: Terminal inverted repeats; TLEs: Tc1-like elements; TSDs: Target site duplicates; UTR: Untranslated region

\section{Acknowledgements}

We are grateful to Dr. Susan R Wessler, the University of Georgia, USA for providing the vectors pWL89a and pAG413gal-ccdB and Dr. David Garfinkel, University of Georgia, USA for the yeast strain DG2523.

\section{Authors' contributions}

MR and MBZ designed the experiments, constructed the TPase expression vector, and wrote the paper; CFP analyzed the location of ECFP fused into NES and estimated the transposition frequency; MR, DQT and KKV analyzed the data; MR, $\mathrm{HH}$ and KKV revised and edited the paper; all authors read and approved the manuscript.

\section{Funding}

This work was funded by a grant from Zhejiang Provincial Natural Science Foundation of China (No. LZ19C160001), the National Natural Science Foundation of China (No 31870656 and 31470615).

\section{Availability of data and materials}

The data generated during this study and datasets supporting the conclusions are included in this article and its Additional file.

\section{Ethics approval and consent to participate}

Not applicable.

\section{Consent for publication}

Not applicable.

\section{Competing interests}

The authors declare that they have no competing interests.

\section{Author details}

'State Key Laboratory of Subtropical Silviculture, Zhejiang A\&F University, Lin'an, Hangzhou 311300, Zhejiang Province, People's Republic of China. ${ }^{2} Z$ hejiang Provincial Collaborative Innovation Center for Bamboo Resources and High-efficiency Utilization, Zhejiang A\&F University, Lin'an, Hangzhou 311300, Zhejiang Province, People's Republic of China. ${ }^{3}$ Division of Genetics, Rice Breeding and Genetics Research Centre, ICAR-Indian Agricultural Research Institute, Aduthurai, Tamil Nadu 612101, India.

Received: 28 March 2019 Accepted: 14 August 2019 Published online: 19 August 2019

\section{References}

1. Feschotte C, Jiang N, Wessler SR. Plant transposable elements: where genetics meets genomics. Nat Rev Genet. 2002;3(5):329-41.

2. Feschotte C, Pritham EJ. DNA transposons and the evolution of eukaryotic genomes. Annu Rev Genet. 2007;41:331-68.

3. Evgen'ev M. Mobile elements and genome evolution. J Mol Biol. 2007:42(2):203-13.

4. Emmons SW, Yesner L, Ruan K-s, Katzenberg D. Evidence for a transposon in Caenorhabditis elegans. Cell. 1983;32(1):55-65.

5. Jacobson JW, Medhora MM, Hartl DL. Molecular structure of a somatically unstable transposable element in Drosophila. Proc Natl Acad Sci U S A. 1986;83(22):8684

6. Hartl DL. Discovery of the transposable element mariner. Genetics. 2001;157(2):471-6.
7. Shao H, Tu Z. Expanding the diversity of the IS630-TC1-mariner superfamily: discovery of a unique DD37E transposon and reclassification of the DD37D and DD39D transposons. Genetics. 2001;159(3):1103-15.

8. Ammar I, Izsvak Z, Ivics Z. The sleeping beauty transposon toolbox. Methods Mol Biol. 2012;859:229-40

9. Robert VJ. Engineering the Caenorhabditis elegans genome by Mos 1induced transgene-instructed gene conversion. Methods Mol Biol. 2012;859:189-201.

10. Tellier M, Bouuaert CC, Chalmers R. Mariner and the ITm superfamily of transposons. Microbiol Spectr. 2015;3(2):Mdna3-0033-2014.

11. Brezinsky L, Wang GV, Humphreys T, Hunt J. The transposable element Uhu from Hawaiian Drosophila--member of the widely dispersed class of Tc1-like transposons. Nucleic Acids Res. 1990;18(8):2053-9.

12. Feschotte C, Wessler SR. Mariner-like transposases are widespread and diverse in flowering plants. Proc Natl Acad Sci U S A. 2002;99(1):280-5.

13. Tudor M, Lobocka M, Goodell M, Pettitt J, O'Hare K. The pogo transposable element family of Drosophila melanogaster. Mol Gen Genet MGG. 1992; 232(1):126-34

14. Lampe DJ, Grant TE, Robertson HM. Factors affecting transposition of the Himar1 mariner transposon in vitro. Genetics. 1998;149(1):179-87.

15. Germon S, Bouchet N, Casteret S, Carpentier G, Adet J, Bigot Y, et al. Mariner Mos 1 transposase optimization by rational mutagenesis. Genetica. 2009; 137(3):265-76.

16. Lampe DJ, Akerley BJ, Rubin EJ, Mekalanos JJ, Robertson HM. Hyperactive transposase mutants of the Himar1 mariner transposon. Proc Natl Acad Sc U S A. 1999:96(20):11428-33.

17. Robertson $\mathrm{H}$. The mariner-like transposable element is widespread in insects. Nature. 1993:362(6417):241-5.

18. Ivics Z, Izsvak Z, Minter A, Hackett PB. Identification of functional domains and evolution of TCl-like transposable elements. Proc Natl Acad Sci U S A. 1996;93(10):5008-13.

19. Fattash I, Lee C-N, Mo K, Yang G. Efficient transposition of the youngest miniature inverted repeat transposable element family of yellow feve mosquito in yeast. FEBS J. 2015:282(10):1829-40.

20. la Cour T, Kiemer L, Molgaard A, Gupta R, Skriver K, Brunak S. Analysis and prediction of leucine-rich nuclear export signals. Protein Eng Des Sel. 2004; 17(6):527-36

21. Xu D, Farmer A, Collett G, Grishin NV, Chook YM. Sequence and structural analyses of nuclear export signals in the NESdb database. Mol Biol Cell. 2012;23(18):3677-93.

22. Demattei M-V, Hedhili S, Sinzelle L, Bressac C, Casteret S, Moiré N, et al. Nuclear importation of mariner transposases among eukaryotes: motif requirements and homo-protein interactions. PLoS One. 2011;6(8):e23693.

23. Lohe AR, De Aguiar D, Hartl DL. Mutations in the mariner transposase: The $D, D(35) E$ consensus sequence is nonfunctional. Proc Natl Acad Sci U S A. 1997;94(4):1293-7

24. Hancock CN, Zhang F, Wessler SR. Transposition of the Tourist-MITE mPing in yeast: an assay that retains key features of catalysis by the class 2 PIF/ Harbinger superfamily. Mob DNA. 2010;1(1):5.

25. Zhou M-B, Lu J-J, Zhong H, Tang K-X, Tang D-Q. Distribution and polymorphism of mariner-like elements in the Bambusoideae subfamily. Plant Syst Evol. 2010;289(1-2):1-11.

26. Zhou M-B, Zhong $\mathrm{H}_{1} \mathrm{Hu}$ J-L, Tang D-O. Ppmarl and Ppmar2: the first two complete and intact full-length mariner-like elements isolated in Phyllostachys edulis. Acta Botanica Gallica. 2015;162(2):127-37.

27. Zhou M, Hu H, Liu Z, Tang D. Two active bamboo mariner-like transposable elements (Ppmar1 and Ppmar2) identified as the transposon-based genetic tools for mutagenesis. Mol Breed. 2016;36(12):163.

28. Zhou MB, Hu H, Miskey C, Lazarow K, Ivics Z, Kunze R, et al. Transposition of the bamboo mariner-like element Ppmar1 in yeast. Mol Phylogenet Evol. 2017;109:367-74.

29. Ramakrishnan M, Zhou M, Pan C, Hanninen H, Yrjala K, Vinod KK, et al. Affinities of terminal inverted repeats to DNA binding domain of transposase affect the transposition activity of bamboo Ppmar2 mariner-like element. Int J Mol Sci. 2019;20(15):3692

30. Fu SC, Huang HC, Horton P, Juan HF. ValidNESs: a database of validated leucine-rich nuclear export signals. Nucleic Acids Res. 2013;41(Database issue):D338-43.

31. Bryksin AV, Matsumura I. Overlap extension PCR cloning: a simple and reliable way to create recombinant plasmids. Biotechniques. 2010; 48(6):463-5. 
32. Anderson Kl, Sanderson J, Gerwig S, Peychl J. A new configuration of the Zeiss LSM 510 for simultaneous optical separation of green and red fluorescent protein pairs. Cytometry A. 2006;69(8):920-9.

33. Schneider CA, Rasband WS, Eliceiri KW. NIH image to ImageJ: 25 years of image analysis. Nat Methods. 2012;9(7):671-5.

34. Michel K, Atkinson P. Nuclear localization of the Hermes transposase depends on basic amino acid residues at the $\mathrm{N}$-terminus of the protein. J Cell Biochem. 2003;89(4):778-90.

35. Bleykasten-Grosshans C, Neuvéglise C. Transposable elements in yeasts. C R Biol. 2011;334(8):679-86.

36. Yang G, Weil CF, Wessler SR. A rice Tc1/mariner-like element transposes in yeast. Plant Cell. 2006;18(10):2469-78.

37. Damelin M, Silver PA. Mapping interactions between nuclear transport factors in living cells reveals pathways through the nuclear pore complex. Mol Cell. 2000;5(1):133-40.

38. Mowen K, David M. Regulation of STAT1 nuclear export by Jak1. Mol Cell Biol. 2000;20(19):7273-81.

39. McBride KM, McDonald C, Reich NC. Nuclear export signal located within the DNA-binding domain of the STAT1transcription factor. EMBO J. 2000; 19(22):6196-206.

40. García-Yagüe Á, Rada P, Rojo Al, Lastres-Becker I, Cuadrado A. Nuclear import and export signals control the subcellular localization of Nurr1 protein in response to oxidative stress. J Biol Chem. 2013;288(8):5506-17.

\section{Publisher's Note}

Springer Nature remains neutral with regard to jurisdictional claims in published maps and institutional affiliations.

Ready to submit your research? Choose BMC and benefit from:

- fast, convenient online submission

- thorough peer review by experienced researchers in your field

- rapid publication on acceptance

- support for research data, including large and complex data types

- gold Open Access which fosters wider collaboration and increased citations

- maximum visibility for your research: over $100 \mathrm{M}$ website views per year

At BMC, research is always in progress.

Learn more biomedcentral.com/submissions 